\title{
Boundary Control of a Flexible Manipulator Based on a High Order Disturbance Observer with Input Saturation
}

\author{
Junteng Ma $\mathbb{D}$, Dongping Jin, Zhengtao Wei, Ti Chen $(\mathbb{D}$, and Hao Wen $(\mathbb{D}$ \\ State Key Laboratory of Mechanics and Control of Mechanical Structures, Nanjing University of Aeronautics and Astronautics, \\ 29 Yudao Street, Nanjing, Jiangsu 210016, China
}

Correspondence should be addressed to Hao Wen; wenhao@nuaa.edu.cn

Received 28 September 2017; Accepted 11 December 2017; Published 8 January 2018

Academic Editor: Evgeny Petrov

Copyright (C) 2018 Junteng Ma et al. This is an open access article distributed under the Creative Commons Attribution License, which permits unrestricted use, distribution, and reproduction in any medium, provided the original work is properly cited.

\begin{abstract}
This paper studies a new boundary control strategy for a flexible manipulator subject to unknown fast time-varying disturbances. The flexible manipulator essentially is an infinite dimensional continuum. Hence, a continuous function of space and time can be employed to describe the position of such a distributed parameter structure, the motion of which can be described by partial differential equations (PDEs). To cope with fast time-varying external disturbances, a high order disturbance observer is adopted. A control strategy based on such a disturbance observer is proposed for the rest-rest maneuvering of the flexible manipulator. Moreover, a smooth hyperbolic function is included in the controller to satisfy the requirement of input saturation. The stability of the boundary control is analyzed using LaSalle's invariance principle. Finally, the performance of the presented boundary controller is verified through comparison with that of employing a constant disturbance observer via numerical simulations.
\end{abstract}

\section{Introduction}

At present, flexible manipulators have increasingly wide applications in industrial, agricultural, medical, and aerospace fields. To meet the demand of higher performance, the trend of development of space manipulators is towards lightweight parts, low energy expenditure, and fast movement $[1,2]$. Since the 1970 s, many studies have been performed on modeling theory and control scheme of flexible manipulators [3]. However, the traditional studies on such distributed parameter structures were based on ordinary differential equations (ODEs) $[4,5]$. Although the ODE dynamic model has a simple form and is convenient for controller design, it is difficult to accurately describe the distributed parameter characteristic of the flexible structure, and spillover instability problems may occur [6]. Compared with the ODE model, the PDE model can reflect the dynamic characteristic of the flexible structure more accurately; however, it will increase the difficulty and challenge of controller design.

Significant attention has been attracted to the PDE modeling and the controller design of flexible manipulators during recent years; that is, the variational principle is allowed to derive the differential equations [7]. For example, Smith obtained the partial differential solutions via the method of finite difference; this work laid the theoretical foundation for the partial differential control method of flexible manipulators [8]. Ge et al. built the PDE model of a distributed parameter flexible manipulator that avoided the traditional modal truncation error [9]. Jiang et al. studied a boundary controller for a flexible arm applying the PDE robust observer [10]. In addition to the studies of straight manipulators, Liu et al. studied the vibration suppression of curved beams using the PDE model [11].

In practice, the performance of the flexible manipulator system is significantly affected by disturbances [12]. To eliminate the influence of disturbances, researchers have developed many solutions, of which the schemes based on disturbance observers are especially effective. The control of the flexible manipulator under distributed disturbances or concentrated disturbances has been discussed in several prior articles. The model of a flexible manipulator was established via PDEs, and an infinite dimensional disturbance observer was applied to estimate the external disturbances in [13]. Morales et al. proposed a nested Generalized Proportional Integral (GPI) controller with a disturbance observer for the 
tip payload changing [14]. The studies with complex disturbances acting on manipulators are also available. Mahamood presented an adaptive hybrid proportional integral derivative control strategy for the two-link flexible manipulator under step signal, square wave signal, white noise disturbance, and sine wave disturbance [15]. Huang et al. studied the motion of an underwater manipulator and developed a disturbance observer for the restoring and coupling forces [16].

Notably, most of the previous studies have investigated the control issue with slowly time-varying disturbances. For example, Chen et al. derived an improved nonlinear disturbance observer that could effectively account for constant disturbances [17]. Yang et al. presented a disturbance observer in the case that the disturbances varied slowly and later defined a lumped disturbance, including model uncertainty, parametric uncertainty, and external disturbances [18]. To optimize trajectory tracking performance, Lee proposed a nonlinear disturbance observer for constant disturbances [19]. In practice, however, the external disturbances are often fast time-varying. To achieve a higher performance, the disturbance observer must be improved. Moreover, it is necessary to consider the condition of input saturation when designing a controller. For example, Liu et al. presented an antiwindup controller for a flexible manipulator in the case of parametric uncertainty, unknown disturbances, and input saturation [20]. Liu et al. investigated the PDE controller of a flexible manipulator with unknown disturbances and input saturation and showed that it worked better than the PD control strategy via numerical simulations [21].

Although the boundary control issue of the flexible manipulator has been discussed extensively, there are few studies on the control problem of flexible manipulators described via PDEs with input saturation and fast timevarying disturbances. Thus, the objectives of this research effort can be summarized as follows: (1) a control law with smooth hyperbolic functions is proposed based on the PDE model, and (2) the use of a higher order disturbance observer to compensate for the fast time-varying disturbances to reduce the disturbance effects.

The organization of this article is as follows: the flexible manipulator is described using PDEs in Section 2; in Section 3, a higher order disturbance observer, is presented to compensate for external disturbances; the control law with input saturation is exhibited in Section 4; numerical simulations are given in Section 5 and a summary of this paper and future perspectives are presented in Section 6.

\section{Dynamic Modeling of the Flexible Manipulator}

The flexible manipulator of concern is shown in Figure 1. In essence, the system is a flexible system that consists of three parts: a motor at the shoulder, a flexible manipulator, and a tip concentrated payload. Because the radius of the motor is extremely small relative to the manipulator, it is ignored in the subsequent analysis.

The manipulator rotates in the horizontal plane at a low speed, driven by the input torque $u(t)$ of the motor. The

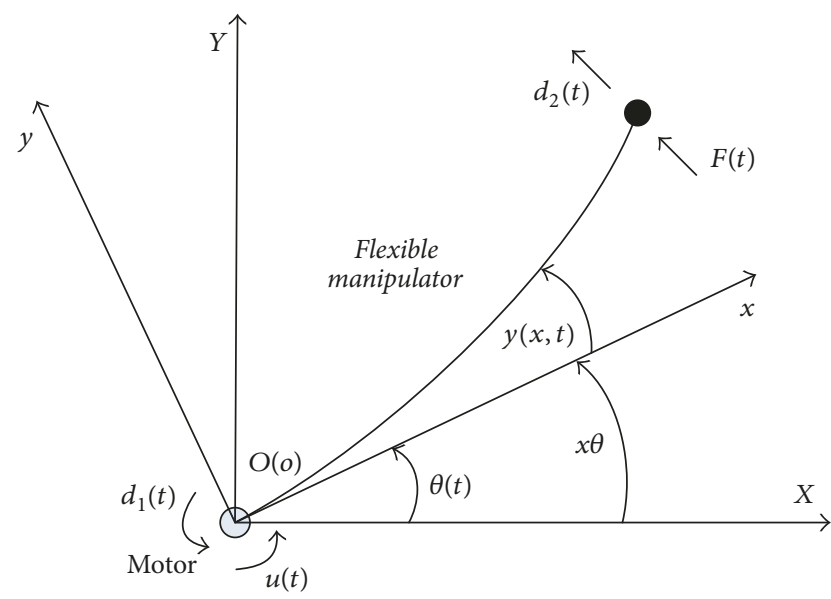

Figure 1: Configuration of a flexible manipulator.

flexible manipulator is subject to small elastic deformation and considered as an Euler-Bernoulli beam. As shown in Figure 1, XOY and xoy are the global frame and the local frame fixed on the body center, respectively; $d_{1}(t)$ and $d_{2}(t)$ denote the input disturbances of the motor and the input disturbances at the tip, respectively; $u(t)$ is the input torque by the motor; $F(t)$ represents the control force acting on the payload; $\theta(t)$ is the rotation angle of the motor; and $y(x, t)$ denotes the elastic deflection at the $x$.

For simplicity, the symbols are introduced as follows:

$$
\begin{aligned}
\theta(t) & =\theta \\
(*)_{x} & =\frac{\partial(*)}{\partial x}, \\
(*)_{x x} & =\frac{\partial^{2}(*)}{\partial x^{2}}, \\
(*)_{x x x} & =\frac{\partial^{3}(*)}{\partial x^{3}} \\
(*)_{x x x x} & =\frac{\partial^{4}(*)}{\partial x^{4}}, \\
(*) & =\frac{\partial(*)}{\partial t}, \\
(*) & =\frac{\partial^{2}(*)}{\partial t^{2}}, \\
(*)^{(3)} & =\frac{\partial^{3}(*)}{\partial t^{3}}, \\
(*)^{(n)} & =\frac{\partial^{n}(*)}{\partial t^{n}}, \quad n=1, \ldots,+\infty .
\end{aligned}
$$

To facilitate the analysis, introduce the auxiliary variable $z(x, t)=x \theta(t)+y(x, t)$. The PDE dynamic model is established by applying Hamilton's principle $\int_{t_{1}}^{t_{2}}\left(\delta E_{k}-\delta E_{p}+\right.$ $\left.\delta W_{c}\right) \mathrm{d} t=0$, where $\delta(\cdot)$ is the variation of $(\cdot) ; \delta E_{k}, \delta E_{p}$, and $\delta W_{c}$ are the variation of the kinetic energy, the potential energy, and the virtual work, respectively [22]. At an arbitrary 
moment, the deflection and the rotation angle are zero at the origin; that is, $y(0, t)=y_{x}(0, t)=0$ such that $z(0, t)=$ $y(0, t)=0, z_{x}(0, t)=\theta, \partial^{n} z / \partial x^{n}=\partial^{n} y / \partial x^{n}(n \geq 2)$.

The total kinetic energy of the flexible manipulator is

$$
E_{k}=\frac{1}{2} I_{h} \dot{\theta}^{2}+\frac{1}{2} \int_{0}^{L} \rho \dot{z}^{2}(x, t) \mathrm{d} x+\frac{1}{2} m \dot{z}^{2}(L, t),
$$

where $I_{h}$ is the rotor inertia, $\rho$ is the mass of per unit length of the flexible manipulator, $m$ is the mass of the tip concentrated payload, and $L$ is the length of the manipulator. The potential energy of the flexible manipulator is

$$
E_{p}=\frac{1}{2} \int_{0}^{L} \operatorname{EI} y_{x x}^{2}(x, t) \mathrm{d} x
$$

where EI is the flexural rigidity.

The nonconservative work is given by

$$
W_{c}=\left[u(t)+d_{1}(t)\right] \theta+\left[F(t)+d_{2}(t)\right] z(L, t) .
$$

Thus, the PDE model of the flexible manipulator reads

$$
\begin{aligned}
& \rho \ddot{z}(x, t)+\mathrm{EI} z_{x x x x}(x, t)=0, \\
& I_{h} \ddot{\theta}-\mathrm{EI} z_{x x}(0, t)-\left[u(t)+d_{1}(t)\right]=0, \\
& m \ddot{z}(L, t)-\mathrm{EI} z_{x x x}(L, t)-\left[F(t)+d_{2}(t)\right]=0, \\
& z_{x x}(L, t)=y_{x x}(L, t)=0, \\
& y(0, t)=y_{x}(0, t)=0 .
\end{aligned}
$$

Two assumptions about the dynamic model are made, as given below.

Assumption 1. The unknown input disturbances $d_{1}$ and $d_{2}$ are bounded; that is, there exist two positive real numbers $D_{1}$ and $D_{2}$ such that $\left|d_{1}\right| \leq D_{1}$ and $\left|d_{2}\right| \leq D_{2}$.

Assumption 2. If the total kinetic energy of the flexible manipulator is bounded for $\forall t \in[0, \infty)$, then both $\dot{\theta}(t)$ and $\dot{z}(x, t)$ are bounded for $\forall(x, t) \in[0, L] \times[0, \infty)$ according to (2) [23].

\section{Disturbance Observer Based Control}

The unknown external disturbances have attracted the attention of many researchers in the field of the control engineering $[24,25]$. The disturbances are induced by not only the system environment but also the uncertainty of the control system, such as the system model uncertainty and parametric uncertainty [24]. Generally, it is difficult to measure the external disturbances accurately; however, a disturbance observer may compensate for the defect.

3.1. Constant Disturbance Observer. Considering the boundary conditions (6) and (7), if the measurements of the state variables $\dot{\theta}$ and $\dot{z}(L, t)$ are available and the initial state conditions $\dot{\theta}_{0}$ and $\dot{z}_{0}$ are known, then the system can be presented as [17]

$$
\begin{aligned}
\ddot{\theta} & =\frac{1}{I_{h}}\left[E I y_{x x}(0, t)+u(t)\right]+\frac{1}{I_{h}} d_{1}(t), \\
\dot{\theta}(0) & =\dot{\theta}_{0}, \\
\ddot{z}(L, t) & =\frac{1}{m}\left[E I y_{x x x}(L, t)+F(t)\right]+\frac{1}{m} d_{2}(t), \\
\dot{z}(L, 0) & =\dot{z}_{0} .
\end{aligned}
$$

Theorem 3. Given two sets of constants $K_{i}>0$ and $K_{j}>$ $0(i, j=1, \ldots, n)$, the constant disturbance observer can be expressed as [17]

$$
\begin{aligned}
& \widehat{d}_{1}=K_{i}\left(I_{h} \dot{\theta}-\gamma_{1}\right), \\
& \dot{\gamma}_{1}=\mathrm{EI} y_{x x}(0, t)+u(t)+\widehat{d}_{1}, \\
& \widehat{d}_{2}=K_{j}\left[m \dot{z}(L, t)-\gamma_{2}\right], \\
& \dot{\gamma}_{2}=\mathrm{EI} y_{x x x}(L, t)+F(t)+\widehat{d}_{2},
\end{aligned}
$$

where $\gamma_{1}$ and $\gamma_{2}$ are the auxiliary variables with $\gamma_{1}(0)=I_{h} \dot{\theta}_{0}$ and $\gamma_{2}(0)=m \dot{z}_{0} ; \widehat{d}_{1}$ and $\widehat{d}_{2}$ are the estimations of $d_{1}$ and $d_{2}$, respectively. Denoting $\widetilde{d}_{1}=d_{1}-\widehat{d}_{1}$ and $\widetilde{d}_{2}=d_{2}-\widehat{d}_{2}$, the disturbance estimation errors $\widetilde{d}_{1}$ and $\widetilde{d}_{2}$ converge to zero exponentially.

Proof. According to the hypothesis $\dot{d}_{1}=0$ and $\dot{d}_{2}=0$, one has $\dot{\tilde{d}}_{1}+K_{i} \tilde{d}_{1}=\dot{d}_{1}=0, \dot{\tilde{d}}_{2}+K_{j} \tilde{d}_{2}=\dot{d}_{2}=0$ from (11), the solutions of which are $\widetilde{d}_{1}=\widetilde{d}_{1}(0) e^{-K_{i} t}, \widetilde{d}_{2}=$ $\widetilde{d}_{2}(0) e^{-K_{j} t}$. Therefore, the disturbance estimation errors $\widetilde{d}_{1}$ and $\widetilde{d}_{2}$ converge to zero exponentially.

3.2. High Order Disturbance Observer. For the fast timevarying disturbances, it is expected that the performance of such constant disturbance observer will deteriorate because the assumption of the slowly varying characteristic on the disturbance is no longer valid [26]. To address the problem, a high order disturbance observer is developed by modeling the unknown time-varying disturbance as [27]

$$
d(t)=\sum_{k=0}^{q} D_{k} t^{k}
$$

where $D_{k}(k \in[0, q])$ are unknown constants. Without the loss of generality, the external disturbance approximated in this work as a quadratic function in time such that

$$
d(t)=D_{0}+D_{1} t+D_{2} t^{2}
$$

Denoting $\widetilde{d}=d-\widehat{d}$, where $\widehat{d}$ and $\widetilde{d}$ are the disturbance estimation and the disturbance tracking error, respectively, the disturbance estimation is constructed as

$$
\widehat{d}(t)=\lambda_{0} h_{0}(t)+\lambda_{1} h_{1}(t)+\lambda_{2} h_{2}(t),
$$


where $\dot{h}_{0}(t)=d-\widehat{d}=\widetilde{d}$ and $h_{k}(t)=\int_{0}^{t} h_{k-1}(\tau) \mathrm{d} \tau$ for $k=$ $1,2,3$. Accordingly, one obtains

$$
\begin{aligned}
\widehat{d}_{1}= & \lambda_{0} \int_{0}^{t} \widetilde{d}_{1}(\tau) \mathrm{d} \tau+\lambda_{1} \int_{0}^{t} \int_{0}^{\delta} \widetilde{d}_{1}(\tau) \mathrm{d} \delta \mathrm{d} \tau \\
& +\lambda_{2} \int_{0}^{t} \int_{0}^{\sigma} \int_{0}^{\delta} \widetilde{d}_{1}(\tau) \mathrm{d} \sigma \mathrm{d} \delta \mathrm{d} \tau, \\
\widehat{d}_{2}= & \lambda_{0} \int_{0}^{t} \widetilde{d}_{2}(\tau) \mathrm{d} \tau+\lambda_{1} \int_{0}^{t} \int_{0}^{\delta} \widetilde{d}_{2}(\tau) \mathrm{d} \delta \mathrm{d} \tau \\
& +\lambda_{2} \int_{0}^{t} \int_{0}^{\sigma} \int_{0}^{\delta} \widetilde{d}_{2}(\tau) \mathrm{d} \sigma \mathrm{d} \delta \mathrm{d} \tau .
\end{aligned}
$$

From (15) and $\mathrm{d} h_{k}(t) / \mathrm{d} t=h_{k-1}$ for $k=1, \ldots, 3$, it can be derived that

$$
\begin{aligned}
& \widehat{\vec{d}}_{1}{ }^{(3)}=\lambda_{0} \ddot{\tilde{d}}_{1}+\lambda_{1} \dot{\tilde{d}}_{1}+\lambda_{2} \tilde{d}_{1}, \\
& \widehat{d}_{2}{ }^{(3)}=\lambda_{0} \ddot{\tilde{d}}_{2}+\lambda_{1} \dot{\tilde{d}}_{2}+\lambda_{2} \tilde{d}_{2} .
\end{aligned}
$$

Substituting $\widetilde{d}_{1}=d_{1}-\widehat{d}_{1}, \widetilde{d}_{2}=d_{2}-\widehat{d}_{2}$ into (16) yields

$$
\begin{aligned}
& d_{1}{ }^{(3)}=\tilde{d}_{1}{ }^{(3)}+\lambda_{0} \ddot{\tilde{d}}_{1}+\lambda_{1} \dot{\vec{d}}_{1}+\lambda_{2} \tilde{d}_{1}, \\
& d_{2}{ }^{(3)}=\widetilde{d}_{2}{ }^{(3)}+\lambda_{0} \ddot{\tilde{d}}_{2}+\lambda_{1} \dot{\vec{d}}_{2}+\lambda_{2} \tilde{d}_{2} .
\end{aligned}
$$

From (13), it can be seen that $d_{1}{ }^{(3)}=0$ and $d_{2}{ }^{(3)}=0$; thus, one has

$$
\begin{aligned}
& \tilde{d}_{1}^{(3)}+\lambda_{0} \ddot{\vec{d}}_{1}+\lambda_{1} \dot{\vec{d}}_{1}+\lambda_{2} \tilde{d}_{1}=d_{1}{ }^{(3)}=0, \\
& \widetilde{d}_{2}{ }^{(3)}+\lambda_{0} \ddot{\widetilde{d}}_{2}+\lambda_{1} \dot{\widetilde{d}}_{2}+\lambda_{2} \widetilde{d}_{2}=d_{2}{ }^{(3)}=0 .
\end{aligned}
$$

Consider the characteristic polynomial

$$
Q(s)=s^{3}+\lambda_{0} s^{2}+\lambda_{1} s+\lambda_{2}
$$

which makes the disturbance error convergent to zero through the Hurwitz stability criterion, if the coefficients of $Q(s)=0$ are chosen properly such that $\lambda_{0}, \lambda_{1}, \lambda_{2}>0$ and $\lambda_{0} \lambda_{1}>\lambda_{2}$.

The auxiliary variables $\dot{\kappa}_{1}$ and $\dot{\kappa}_{2}$ are defined as

$$
\begin{aligned}
& \dot{\kappa}_{1}=\mathrm{EI} y_{x x}(0, t)+u+\widehat{d}_{1}, \\
& \dot{\kappa}_{2}=\mathrm{EI} y_{x x x}(L, t)+F+\widehat{d}_{2},
\end{aligned}
$$

where $\kappa_{1}(0)=I_{h} \dot{\theta}_{0}$ and $\kappa_{2}(0)=m \dot{z}_{0}$.

Combining (6) and (7) with (20), one obtains

$$
\begin{aligned}
I_{h} \ddot{\theta}-\dot{\kappa}_{1} & =I_{h} \ddot{\theta}-\left[\mathrm{EI} y_{x x}(0, t)+u+\widehat{d}_{1}\right] \\
& =d_{1}-\widehat{d}_{1}=\widetilde{d}_{1}, \\
m \ddot{z}(L, t)-\dot{\kappa}_{2} & =m \ddot{z}(L, t)-\left[\operatorname{EI} y_{x x x}(L, t)+F+\widehat{d}_{2}\right] \\
& =d_{2}-\widehat{d}_{2}=\tilde{d}_{2} .
\end{aligned}
$$

Integrating both sides from 0 to $t$ yields

$$
\begin{array}{r}
I_{h} \dot{\theta}-\kappa_{1}=\int_{0}^{t} \tilde{d}_{1} \mathrm{~d} \tau, \\
m \dot{z}(L, t)-\kappa_{2}=\int_{0}^{t} \tilde{d}_{2} \mathrm{~d} \tau .
\end{array}
$$

By observing (15), (20), and (22), the high order disturbance observers for the flexible manipulator can be formulated as follows:

$$
\begin{aligned}
\widehat{d}_{1}= & \lambda_{0}\left(I_{h} \dot{\theta}-\kappa_{1}\right)+\lambda_{1} \int_{0}^{t}\left(I_{h} \dot{\theta}-\kappa_{1}\right) \mathrm{d} \tau \\
& +\lambda_{2} \int_{0}^{t} \int_{0}^{\delta}\left(I_{h} \dot{\theta}-\kappa_{1}\right) \mathrm{d} \delta \mathrm{d} \tau, \\
\dot{\kappa}_{1}= & \operatorname{EI} y_{x x}(0, t)+u+\widehat{d}_{1}, \\
\widehat{d}_{2}= & \lambda_{0}\left[m \dot{z}(L, t)-\kappa_{2}\right]+\lambda_{1} \int_{0}^{t}\left[m \dot{z}(L, t)-\kappa_{2}\right] \mathrm{d} \tau \\
& +\lambda_{2} \int_{0}^{t} \int_{0}^{\delta}\left[m \dot{z}(L, t)-\kappa_{2}\right] \mathrm{d} \delta \mathrm{d} \tau, \\
\dot{\kappa}_{2}= & \operatorname{EI} y_{x x x}(L, t)+F+\widehat{d}_{2},
\end{aligned}
$$

where $\lambda_{0}, \lambda_{1}, \lambda_{2}>0$ and $\lambda_{0} \lambda_{1}>\lambda_{2}$.

\section{Controller Design and Analysis}

4.1. Control Scheme. The objective of the scheme is to drive the angle of the motor at the shoulder to the desired value and realize the vibration suppression of the elastic beam simultaneously. By employing the high order disturbance observer, the control laws with input saturation are constructed as follows:

$$
\begin{aligned}
u= & -\alpha_{1} l_{1} \tanh \left(l_{1} e\right)-\alpha_{2} l_{2} \tanh \left(l_{2} \dot{e}\right)-k \operatorname{sgn}(\dot{e}) \\
& -\widehat{d}_{1}, \\
F= & -\alpha_{3} l_{3} \tanh \left[l_{3} \dot{z}(L, t)\right]-k \operatorname{sgn}[\dot{z}(L, t)]-\widehat{d}_{2},
\end{aligned}
$$

where $\alpha_{1}, \alpha_{2}, \alpha_{3}, l_{1}, l_{2}$, and $l_{3}>0 ; k \geq \max \left(\left|\tilde{d}_{1}\right|_{\max },\left|\tilde{d}_{2}\right|_{\max }\right)$; $e=\theta-\theta_{d}, \dot{e}=\dot{\theta}, \ddot{e}=\ddot{\theta}$, and $\theta_{d}$ is the desired angle.

Theorem 4. The control laws are given by (24) with the high order disturbance observer.

(1) The boundary control is asymptotically stable: for $x \in$ $[0, L]$ one has $\theta \rightarrow \theta_{d}, \dot{\theta} \rightarrow 0, y(x, t) \rightarrow 0, \dot{y}(x, t) \rightarrow 0$, $\dot{z}(L, t) \rightarrow 0, \widetilde{d}_{1} \rightarrow 0, \tilde{d}_{2} \rightarrow 0$ when $t \rightarrow \infty$.

(2) The inputs are bounded, such as

$$
\begin{aligned}
& |u| \leq \alpha_{1} l_{1}+\alpha_{2} l_{2}+k+\bar{D}_{1}, \\
& |F| \leq \alpha_{3} l_{3}+k+\bar{D}_{2} .
\end{aligned}
$$

Proof. (1) See Appendix for the details of the proof.

(2) For arbitrary $x$, the domain of the hyperbolic tangent function $y=\tanh (x)$ is $[-1,1]$. For $x$ increasing from 0 to 
TABLE 1: Physical parameters of the manipulator.

\begin{tabular}{lcc}
\hline Parameter & Physical significance & Value \\
\hline EI $\left(\mathrm{N} \cdot \mathrm{m}^{2}\right)$ & Flexural rigidity of the beam & 2 \\
$\rho(\mathrm{kg} / \mathrm{m})$ & Mass of per unit length & 0.2 \\
$I_{h}\left(\mathrm{~kg} \cdot \mathrm{m}^{2}\right)$ & Inertia of the motor & 0.5 \\
$L(\mathrm{~m})$ & Length of the flexible beam & 1 \\
$m(\mathrm{~kg})$ & $\quad$ Mass at the tip & 2 \\
\hline
\end{tabular}

$+\infty, y$ increases very rapidly at the beginning, and finally $y \rightarrow 1$. According to the property of the odd function $\tanh (x), y \rightarrow-1$ for $x$ decreasing from 0 to $-\infty$. Therefore, the hyperbolic tangent function can well simulate the process of input saturation, and the absolute value $|\tanh (x)| \leq 1$.

Based on the above analysis, one has $\left|-\alpha_{1} l_{1} \tanh \left(l_{1} e\right)\right| \leq$ $\left|-\alpha_{1} l_{1}\right|=\alpha_{1} l_{1},\left|-\alpha_{2} l_{2} \tanh \left(l_{2} e\right)\right| \leq\left|-\alpha_{2} l_{2}\right|=\alpha_{2} l_{2}$, and $\left|-\alpha_{3} l_{3} \tanh \left[l_{3} \dot{z}(L, t)\right]\right| \leq\left|-\alpha_{3} l_{3}\right|=\alpha_{3} l_{3}$. As is known, $|k \operatorname{sgn}(\dot{e})| \leq k,\left|\widehat{d}_{1}\right| \leq \bar{D}_{1}$, and $\left|\widehat{d}_{2}\right| \leq \bar{D}_{2}$; therefore,

$|u|$

$$
\begin{aligned}
& =\left|-\alpha_{1} l_{1} \tanh \left(l_{1} e\right)-\alpha_{2} l_{2} \tanh \left(l_{2} \dot{e}\right)-k \operatorname{sgn}(\dot{e})-\widehat{d}_{1}\right| \\
& \leq \alpha_{1} l_{1}+\alpha_{2} l_{2}+k+\bar{D}_{1}, \\
& |F|=\left|-\alpha_{3} l_{3} \tanh \left[l_{3} \dot{z}(L, t)\right]-k \operatorname{sgn}[\dot{z}(L, t)]-\widehat{d}_{2}\right| \\
& \leq \alpha_{3} l_{3}+k+\bar{D}_{2},
\end{aligned}
$$

where $\bar{D}_{1}$ and $\bar{D}_{2}$ denote the boundaries of disturbance estimations. The above $|u|$ and $|F|$ can satisfy the condition of input saturation by changing the parameters $\alpha_{1}, \alpha_{2}, \alpha_{3}, l_{1}$, $l_{2}, l_{3}$, and $k$.

\section{Numerical Simulation Examples}

Numerical simulations are presented in this section to verify the boundary control based on the high order disturbance observer with input saturation. For comparison purposes, the simulations under the control scheme employing the constant disturbance observer are also given in this section. The physical parameters of the manipulator are listed in Table 1.

The aim of all the simulations is to drive the angle of the motor to the desired value; that is, $\theta_{d}=0.5 \mathrm{rad}$, without residual vibration of the elastic beam. The external disturbances are hybrid wave signals.

The parameters in the control scheme proposed in this paper are chosen as $\alpha_{1}=80, \alpha_{2}=10, \alpha_{3}=100, l_{1}=0.4$, $l_{2}=1, l_{3}=0.2, k=0.075, \lambda_{0}=120, \lambda_{1}=60$, and $\lambda_{2}=60$.

In the control strategy employing the constant disturbance observer, the parameters are chosen as $\alpha_{1}=80, \alpha_{2}=$ $10, \alpha_{3}=100, l_{1}=0.4, l_{2}=1, l_{3}=0.2, K_{i}=5$, and $K_{j}=5$.

As indicated in Figure 2(a), under the proposed control scheme, the angle of the motor arrives at the specified location in approximately 12 seconds and later stops steadily. The response curves in Figure 2(a) are quite smooth in shape. However, under the control scheme employing the constant disturbance observer, Figure 2(b) shows that there exist significant positioning errors, even in the last part of the angular response.

Figure 3 depicts the deflections at the tip and at the middle of the flexible manipulator. As shown in Figure 3(a), the amplitude of the elastic vibration under the proposed control law with the high order observer gradually decreases to zero in 12 seconds. By contrast, under the control scheme employing the constant disturbance observer, the residual vibration of the flexible manipulator does not vanish, even after a long time.

The disturbance estimates by the two types of disturbance observers are compared in Figures 4 and 5 (zoom-in view). The simulation results indicate that the high order disturbance observer outperforms the constant one in terms of the disturbance estimation errors, especially for the disturbances of fast time-varying or transient. The constant disturbance observer leads to a relatively poor performance in the scenario and is suitable to estimate the slow time-varying disturbances or smooth signals.

\section{Conclusions}

The motion of the flexible manipulator was described via PDEs to overcome the problems caused by model truncation, and a high order disturbance observer was proposed thereafter to estimate the external disturbances for counteracting the disturbance effects. The physical requirement of input saturation was considered in the proposed control law using smooth hyperbolic functions. The stability of the boundary control system was demonstrated using LaSalle's invariance principle. Finally, numerical simulations illustrated that the boundary controller works notably well. By contrast with the constant disturbance observer, the high order disturbance observer can accurately estimate the fast time-varying disturbances or the transient signals. In the future, the vibration suppression of multilink flexible manipulators will be discussed.

\section{Appendix}

\section{Demonstration of Theorem 4(1)}

Proof. The parameters $\dot{\theta}, \dot{z}(L, t), y_{x x}(0, t)$, and $y_{x x x}(L, t)$ can be measured by the experiment instrument.

The Lyapunov function is defined as

$$
V=E_{1}+E_{2}+E_{3}
$$



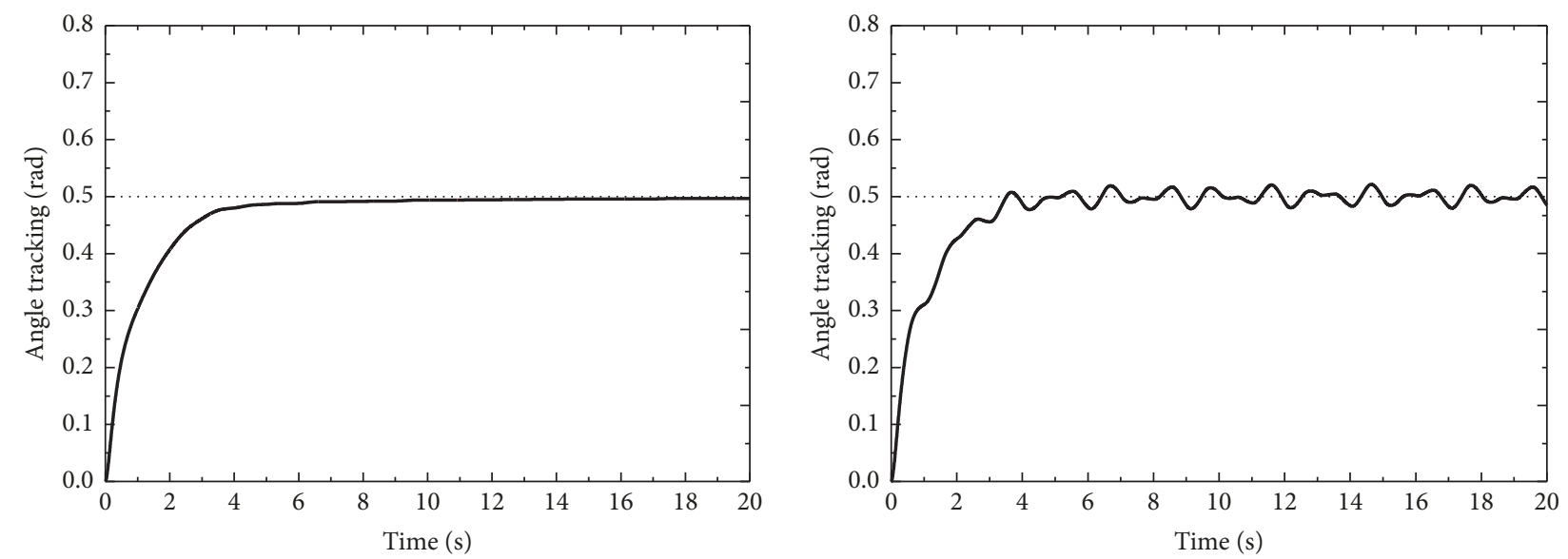

$\begin{array}{ll}- & \theta \\ \cdots & \theta_{d}\end{array}$

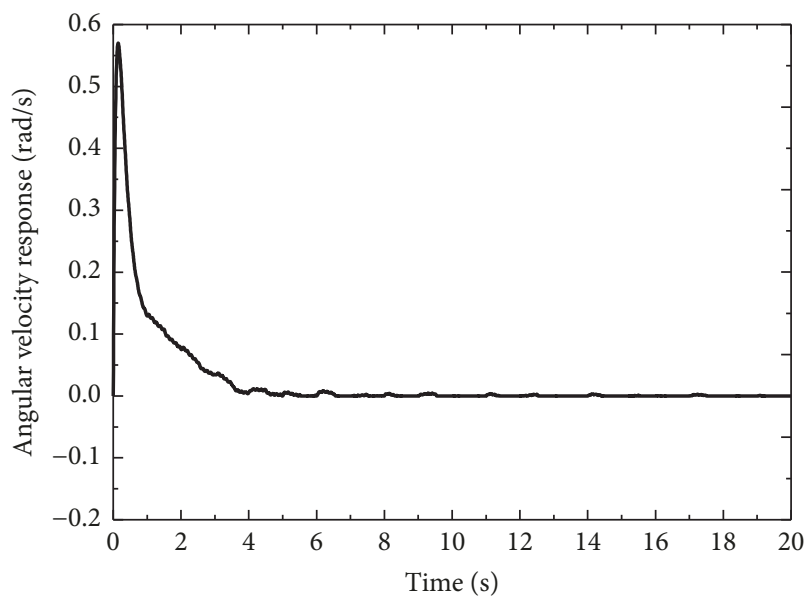

(a)

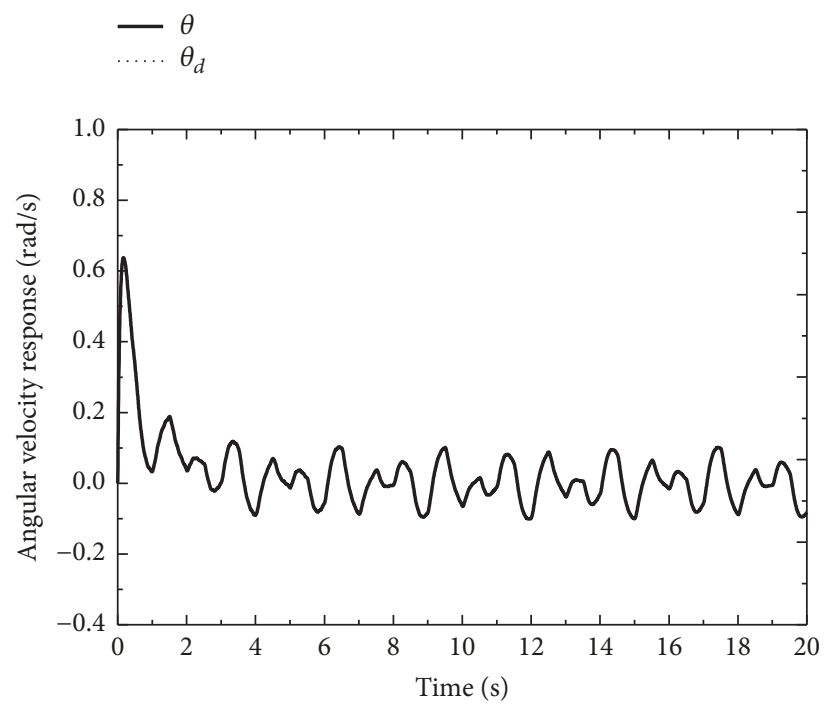

(b)

FIGURE 2: Responses in motor angle and angular velocity for (a) the boundary control scheme with the high order observer and (b) the control scheme employing the constant disturbance observer.

where

$$
\begin{aligned}
E_{1}= & \frac{1}{2} \int_{0}^{L} \rho \dot{z}^{2}(x, t) \mathrm{d} x+\frac{1}{2} \mathrm{EI} \int_{0}^{L} y_{x x}^{2}(x, t) \mathrm{d} x \\
E_{2}= & \frac{1}{2} I_{h} \dot{e}^{2}+\alpha_{1} \ln \left[\cosh \left(l_{1} e\right)\right]+\frac{1}{2} m \dot{z}^{2}(L, t) \\
E_{3}= & \lambda_{2} \lambda_{0}{ }^{2} \widetilde{d}_{1}^{2}+2 \lambda_{2} \lambda_{0} \tilde{d}_{1} \dot{\tilde{d}}_{1}+\left(\lambda_{1} \lambda_{0}+\lambda_{0}{ }^{3}\right) \dot{\tilde{d}}_{1}^{2} \\
& +2 \lambda_{0}{ }^{2} \dot{\tilde{d}}_{1} \ddot{\tilde{d}}_{1}+\lambda_{0} \ddot{\tilde{d}}_{1}^{2}+\lambda_{2} \lambda_{0}{ }^{2} \widetilde{d}_{2}^{2} \\
& +2 \lambda_{2} \lambda_{0} \tilde{d}_{2} \dot{\tilde{d}}_{2}+\left(\lambda_{1} \lambda_{0}+\lambda_{0}^{3}\right) \dot{\tilde{d}}_{2}^{2} \\
& +2 \lambda_{0}{ }^{2} \dot{\tilde{d}}_{2} \ddot{\tilde{d}}_{2}+\lambda_{0} \ddot{\tilde{d}}_{2}^{2}
\end{aligned}
$$

where $E_{1}$ represents the sum of the kinetic energy and the elastic energy of the manipulator, $E_{2}$ represents the payload energy and the control error index, and $E_{3}$ represents the error of the observer.

According to hypothesis (18),

$$
\dot{V}=\dot{E}_{1}+\dot{E}_{2}+\dot{E}_{3}
$$

where

$$
\begin{aligned}
\dot{E}_{1} & =\int_{0}^{L} \rho \dot{z}(x, t) \ddot{z}(x, t) \mathrm{d} x \\
& +\mathrm{EI} \int_{0}^{L} y_{x x}(x, t) \dot{y}_{x x}(x, t) \mathrm{d} x \\
& =-\mathrm{EI} \int_{0}^{L} \dot{z}(x, t) z_{x x x x}(x, t) \mathrm{d} x \\
& +\mathrm{EI} \int_{0}^{L} y_{x x}(x, t) \dot{y}_{x x}(x, t) \mathrm{d} x \\
& =-\mathrm{EI}\left[\dot{z}(L, t) z_{x x x}(L, t)\right.
\end{aligned}
$$



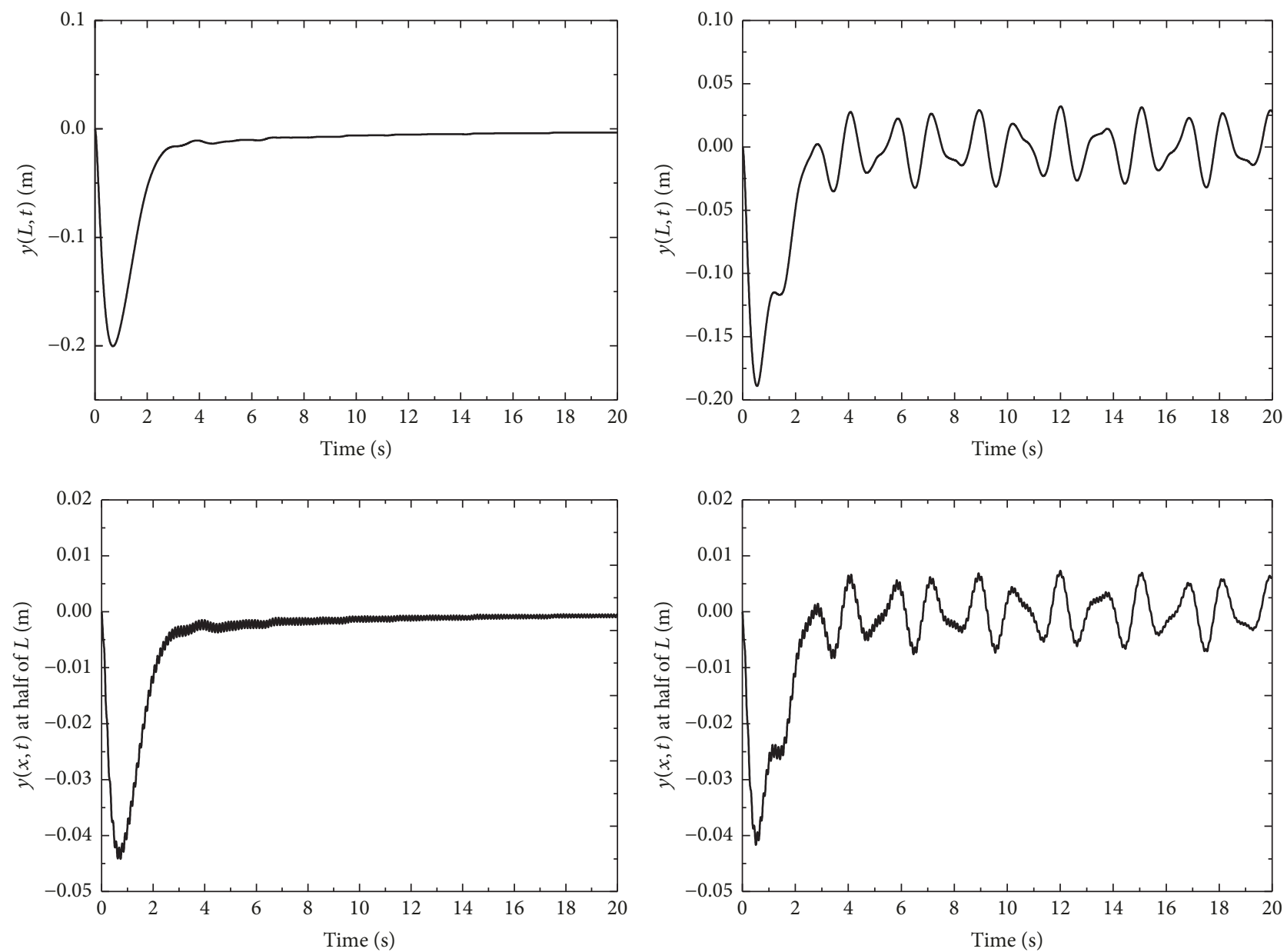

(a)

(b)

Figure 3: Deflections at the tip and at the middle for (a) the boundary control scheme with the high order observer and (b) the control scheme employing the constant disturbance observer.

$$
\begin{aligned}
- & \left.\int_{0}^{L} \dot{z}_{x}(x, t) z_{x x x}(x, t) \mathrm{d} x\right]+\mathrm{EI}\left[-z_{x x}(0, t) \dot{\theta}\right. \\
& \left.-\int_{0}^{L} \dot{z}_{x}(x, t) z_{x x x}(x, t) \mathrm{d} x\right]=-\mathrm{EI} \dot{z}(L, t) \\
& \cdot y_{x x x}(L, t)-\mathrm{EI} y_{x x}(0, t) \dot{\theta}, \\
\dot{E}_{2}= & I_{h} \ddot{e} \ddot{e}+\alpha_{1} l_{1} \tanh \left(l_{1} e\right) \dot{e}+m \dot{z}(L, t) \ddot{z}(L, t) \\
& =\dot{e}\left[I_{h} \ddot{e}+\alpha_{1} l_{1} \tanh \left(l_{1} e\right)\right]+m \dot{z}(L, t) \ddot{z}(L, t), \\
\dot{E}_{3}= & \left(2 \lambda_{0} \lambda_{2}-2 \lambda_{1} \lambda_{0}{ }^{2}\right) \dot{\tilde{d}}_{1}^{2}+\left(2 \lambda_{0} \lambda_{2}\right. \\
& \left.-2 \lambda_{1} \lambda_{0}{ }^{2}\right) \dot{\tilde{d}}_{2}^{2},
\end{aligned}
$$

where

$$
\begin{aligned}
\dot{V} & =\dot{E}_{1}+\dot{E}_{2}+\dot{E}_{3} \\
& =\dot{z}(L, t)\left[F+d_{2}-m \ddot{z}(L, t)\right]
\end{aligned}
$$

$$
\begin{aligned}
+ & \dot{e}\left[I_{h} \ddot{e}+\alpha_{1} l_{1} \tanh \left(l_{1} e\right)-\operatorname{EI} y_{x x}(0, t)\right] \\
+ & m \dot{z}(L, t) \ddot{z}(L, t)+\left(2 \lambda_{0} \lambda_{2}-2 \lambda_{1} \lambda_{0}{ }^{2}\right) \dot{\tilde{d}}_{1}^{2} \\
& +\left(2 \lambda_{0} \lambda_{2}-2 \lambda_{1} \lambda_{0}^{2}\right) \dot{\tilde{d}}_{2}^{2} \\
= & \dot{z}(L, t)\left(F+d_{2}\right)+\dot{e}\left[u+d_{1}+\alpha_{1} l_{1} \tanh \left(l_{1} e\right)\right] \\
& +\left(2 \lambda_{0} \lambda_{2}-2 \lambda_{1} \lambda_{0}{ }^{2}\right) \dot{\tilde{d}}_{1}^{2} \\
& +\left(2 \lambda_{0} \lambda_{2}-2 \lambda_{1} \lambda_{0}{ }^{2}\right) \dot{\tilde{d}}_{2}^{2} \\
= & \dot{e}\left[u+d_{1}+\alpha_{1} l_{1} \tanh ^{2}\left(l_{1} e\right)\right]+\dot{z}(L, t)\left(F+d_{2}\right) \\
& +\left(2 \lambda_{0} \lambda_{2}-2 \lambda_{1} \lambda_{0}{ }^{2}\right) \dot{\tilde{d}}_{1}^{2} \\
& +\left(2 \lambda_{0} \lambda_{2}-2 \lambda_{1} \lambda_{0}{ }^{2}\right) \dot{\tilde{d}}_{2}^{2} \\
= & -\alpha l_{2} \dot{e} \tanh \left(l_{2} \dot{e}\right)-\alpha_{3} l_{3} \dot{z}(L, t) \tanh \left[l_{3} \dot{z}(L, t)\right]
\end{aligned}
$$



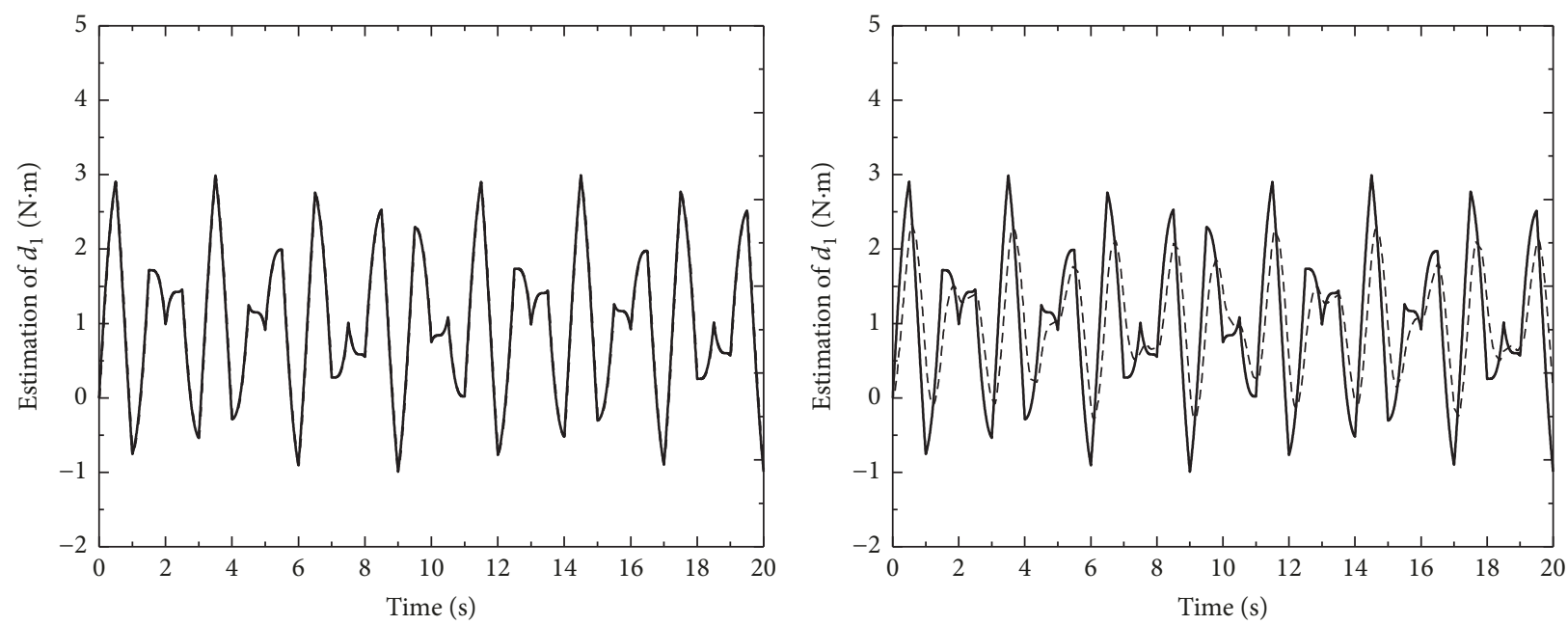

$-d_{1}$

- - $d_{1}$ estimation

$-d_{1}$

$d_{1}$ estimation

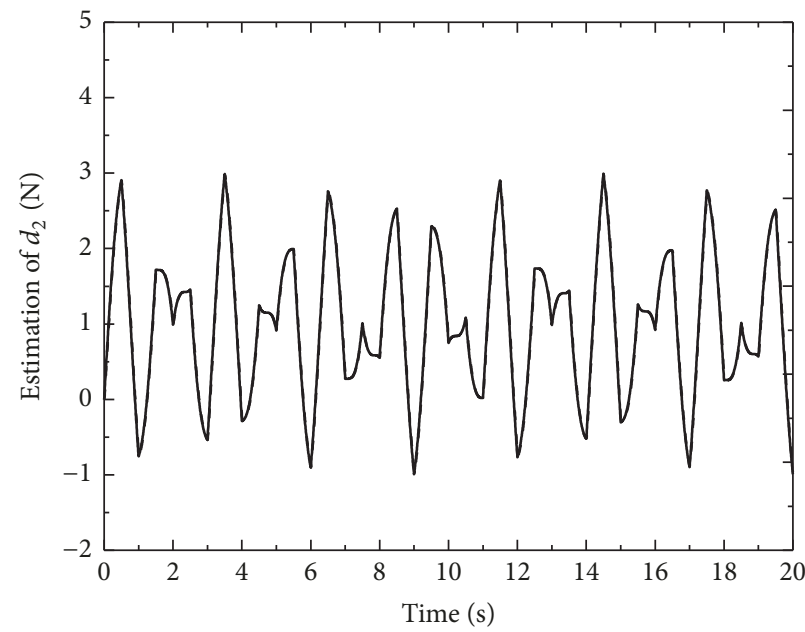

$-d_{2}$

- - $d_{2}$ estimation

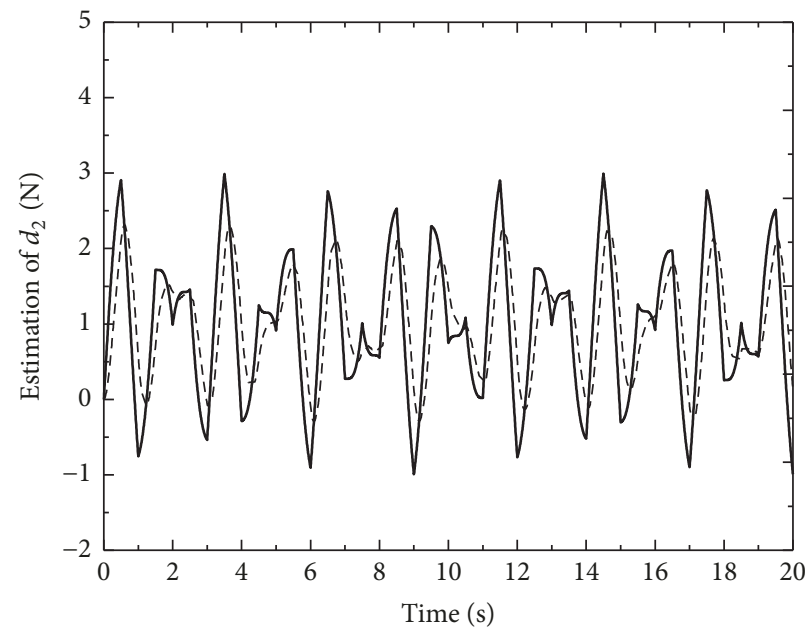

$-d_{2}$

- - $d_{2}$ estimation

(a)

(b)

Figure 4: Disturbance estimates for (a) the boundary control scheme with the high order observer and (b) the control scheme employing the constant disturbance observer.

$$
\begin{aligned}
& +\left(\widetilde{d}_{1} \dot{e}-k|\dot{e}|\right)+\left[\widetilde{d}_{2} \dot{z}(L, t)-k|\dot{z}(L, t)|\right] \\
& +\left(2 \lambda_{0} \lambda_{2}-2 \lambda_{1} \lambda_{0}{ }^{2}\right) \dot{\tilde{d}}_{1}^{2} \\
& +\left(2 \lambda_{0} \lambda_{2}-2 \lambda_{1} \lambda_{0}{ }^{2}\right) \dot{\tilde{d}}_{2}{ }^{2} .
\end{aligned}
$$

Because $x \tanh (x) \geq 0, \lambda_{0}, \lambda_{1}, \lambda_{2}>0, \lambda_{0} \lambda_{1}>\lambda_{2}$, and $k \geq$ $\max \left(\left|\tilde{d}_{1}\right|_{\max },\left|\tilde{d}_{2}\right|_{\max }\right)$, thus $\dot{V} \leq 0$.

LaSalle's invariance principle is applied to analyze the stability of the controller $(\dot{V} \equiv 0 \rightarrow y(x, t) \equiv 0)$.
The stability of the controller when $\dot{V} \equiv 0$ is verified as follows:

$$
\begin{aligned}
& \dot{V} \equiv 0 \longrightarrow \\
& \dot{e} \equiv \dot{z}(L, t) \equiv \dot{\tilde{d}}_{1} \equiv \dot{\tilde{d}}_{2} \equiv 0 ;
\end{aligned}
$$

thus, it can be seen that

$$
\begin{aligned}
& \ddot{e}=\ddot{z}(L, t)=0 \longrightarrow \\
& \dot{\theta} \equiv \ddot{\theta} \equiv \ddot{\bar{d}}_{1} \equiv \ddot{\bar{d}}_{2} \equiv \widetilde{d}_{1}^{(3)} \equiv \widetilde{d}_{2}^{(3)} \equiv 0 .
\end{aligned}
$$



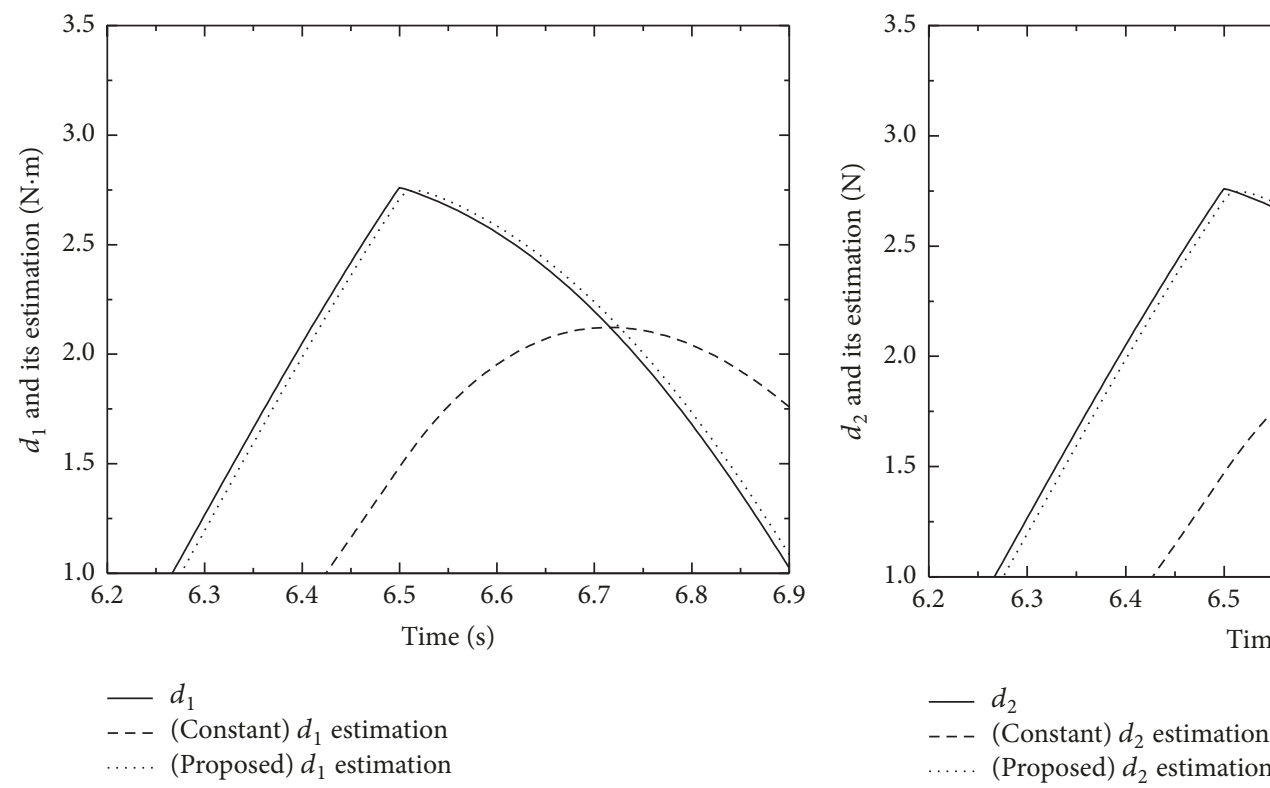

- - $d_{1}$ (Constant) $d_{1}$ estimation ..... (Proposed) $d_{1}$ estimation

- - - (Constant) $d_{2}$ estimation (Proposed) $d_{2}$ estimation

FIGURE 5: Comparison of the disturbance estimations.

According to (18), one obtains

$$
\tilde{d}_{1} \equiv \tilde{d}_{2} \equiv 0 .
$$

Substituting $\ddot{z}(x, t)=x \ddot{\theta}(t)+\ddot{y}(x, t)$ into $\rho \ddot{z}(x)=$ $-\mathrm{EI} z_{x x x x}(x)$ yields

$$
\rho \ddot{y}(x, t)=-\mathrm{EI} y_{x x x x}(x, t) ;
$$

thus

$$
\begin{aligned}
\rho \ddot{z}(L, t) & =-\mathrm{EI} y_{x x x x}(L, t)=0 \longrightarrow \\
y_{x x x x}(L, t) & =0 .
\end{aligned}
$$

The variable separation method is adopted as

$$
\begin{aligned}
y(x, t) & =X(x) T(t), \\
\rho \ddot{y}(x, t) & =-\mathrm{EI} y_{x x x x}(x, t) \longrightarrow \\
y_{x x x x}(x, t) & =-\frac{\rho}{\mathrm{EI}} \ddot{y}(x, t), \\
y_{x x x x}(x, t) & =X^{(4)}(x) \cdot T(t), \\
\ddot{y}(x, t) & =X(x) \cdot \ddot{T}(t) \\
& \downarrow \\
\frac{X^{(4)}(x)}{X(x)} & =-\frac{\rho}{\mathrm{EI}} \frac{\ddot{T}(t)}{T(t)}=\mu \\
& \downarrow \\
X^{(4)}(x)-\mu X(x) & =0 .
\end{aligned}
$$

Letting $\mu=s^{4}$, the solution of (A.13) is

$$
\begin{aligned}
X(x)= & c_{1} \cosh s x+c_{2} \sinh s x+c_{3} \cos s x \\
& +c_{4} \sin s x .
\end{aligned}
$$

Because $y(0, t)=0, y_{x}(0, t)=0, y_{x x}(L, t)=0$, and $y_{x x x x}(L, t)=0$, the following can be obtained:

$$
X(0)=X_{x}(0)=X_{x x}(L)=X_{x x x x}(L)=0 .
$$

Then

$$
\begin{aligned}
c_{1}+c_{3} & =0, \\
c_{2}+c_{4} & =0, \\
c_{1} \cosh s L+c_{2} \sinh s L-c_{3} \cos s L-c_{4} \sin s L & =0, \\
c_{1} \cosh s L+c_{2} \sinh s L+c_{3} \cos s L+c_{4} \sin s L & =0 ;
\end{aligned}
$$

the equations are simplified as $c_{4}(\sinh s L \cdot \cos s L-\sin s L$. $\cosh s L)=0$, and the solutions are $c_{i}=0(i=1,2,3,4)$ for all $s$. Accordingly, one obtains $X(x)=0, y(x, t)=0, \dot{y}(x, t)=0$, and $y_{x x}(0, t)=0$.

Because $z_{x x}(0, t)=y_{x x}(0, t)=0$, by observing (A.6), (A.7), (A.8), and

$$
\begin{aligned}
& I_{h} \ddot{\theta}-\mathrm{EI} z_{x x}(0, t)-\left[u(t)+d_{1}(t)\right]=0, \\
& \text { u } \\
& \begin{aligned}
= & -\alpha_{1} l_{1} \tanh \left(l_{1} e\right)-\alpha_{2} l_{2} \tanh \left(l_{2} \dot{e}\right)-k \operatorname{sgn}(\dot{e}) \\
& -\widehat{d}_{1},
\end{aligned}
\end{aligned}
$$

the following can be obtained: $-\alpha_{1} l_{1} \tanh \left(l_{1} e\right)+\tilde{d}_{1}=0 \rightarrow e=$ 0 .

Therefore, the PDE boundary control in this paper is asymptotically stable by applying the extended LaSalle's invariance principle; that is, for $x \in[0, L]$ one has $\theta \rightarrow \theta_{d}$, $\dot{\theta} \rightarrow 0, y(x, t) \rightarrow 0, \dot{y}(x, t) \rightarrow 0, \dot{z}(L, t) \rightarrow 0, \widetilde{d}_{1} \rightarrow 0$, $\tilde{d}_{2} \rightarrow 0$, when $t \rightarrow \infty$. 


\section{Conflicts of Interest}

The authors declare that there are no conflicts of interest regarding the publication of this paper.

\section{Acknowledgments}

This work was supported by the National Natural Science Foundation of China (Project nos. 11372130 and 11290153), the Equipment Pre-Research Foundation (Grant no. 6140210010202), and the Research Fund of the State Key Laboratory of Mechanics and Control of Mechanical Structures (Nanjing University of Aeronautics and Astronautics) (Grant no. MCMS-0116K01).

\section{References}

[1] M. Shan, J. Guo, and E. Gill, "Review and comparison of active space debris capturing and removal methods," Progress in Aerospace Sciences, vol. 80, pp. 18-32, 2016.

[2] Z. Yu, Q. Shi, H. Wang, N. Yu, Q. Huang, and T. Fukuda, "How to achieve precise operation of a robotic manipulator on a macro to micro/nano scale," Assembly Automation, vol. 37, no. 2, pp. 186-199, 2017.

[3] J. C. Ower and J. van de Vegte, "Classical Control Design for a Flexible Manipulator: Modeling and Control System Design," IEEE Journal on Robotics and Automation, vol. 3, no. 5, pp. 485489, 1987.

[4] H. Yang, Y. Yu, Y. Yuan, and X. Fan, "Back-stepping control of two-link flexible manipulator based on an extended state observer," Advances in Space Research, vol. 56, no. 10, pp. 23122322, 2015.

[5] H. Wen, T. Chen, D. Jin, and H. Hu, "Passivity-based control with collision avoidance for a hub-beam spacecraft," Advances in Space Research, vol. 59, no. 1, pp. 425-433, 2017.

[6] T. Chen, H. Wen, H. Hu, and D. Jin, "Quasi-time-optimal controller design for a rigid-flexible multibody system via absolute coordinate-based formulation," Nonlinear Dynamics, vol. 88, no. 1, pp. 623-633, 2017.

[7] K. W. Cassel, Variational Methods with Applications in Science and Engineering, Cambridge University Press, 2013.

[8] G. D. Smith, Numerical Solution of Partial Differential Equation: Finite Difference Methods, Oxford University Press, 1971.

[9] S. S. Ge, T. H. Lee, G. Zhu, and F. Hong, "Variable structure control of a distributed-parameter flexible beam," Journal of Robotic Systems, vol. 18, no. 1, pp. 17-27, 2001.

[10] T. Jiang, J. Liu, and W. He, "Boundary control for a flexible manipulator with a robust state observer," Journal of Vibration and Control, pp. 1-12, 2016.

[11] F. Liu, D. Jin, and H. Wen, "Optimal vibration control of curved beams using distributed parameter models," Journal of Sound and Vibration, vol. 384, pp. 15-27, 2016.

[12] Y. Zhang, M. Li, and J. Zhang, "Vibration Control for Rapid Attitude Stabilization of Spacecraft," IEEE Transactions on Aerospace and Electronic Systems, vol. 53, no. 3, pp. 1308-1320, 2017.

[13] T. Jiang, J. Liu, and W. He, "Boundary control for a flexible manipulator based on infinite dimensional disturbance observer," Journal of Sound and Vibration, vol. 348, pp. 1-14, 2015.
[14] R. Morales, V. Feliu, and V. Jaramillo, "Position control of very lightweight single-link flexible arms with large payload variations by using disturbance observers," Robotics and Autonomous Systems, vol. 60, no. 4, pp. 532-547, 2012.

[15] R. M. Mahamood, Adaptive Controller Design for Two-Link Flexible Manipulator, Springer Netherlands, 2014.

[16] H. Huang, Q. Tang, H. Li, L. Liang, W. Li, and Y. Pang, "Vehicle-manipulator system dynamic modeling and control for underwater autonomous manipulation," Multibody System Dynamics, vol. 41, no. 2, pp. 125-147, 2017.

[17] W.-H. Chen, D. J. Ballance, P. J. Gawthrop, and J. O'Reilly, "A nonlinear disturbance observer for robotic manipulators," IEEE Transactions on Industrial Electronics, vol. 47, no. 4, pp. 932-938, 2000.

[18] J. Yang, W.-H. Chen, and S. Li, "Non-linear disturbance observer-based robust control for systems with mismatched disturbances/uncertainties," IET Control Theory \& Applications, vol. 5, no. 18, pp. 2053-2062, 2011.

[19] D. Lee, "Nonlinear disturbance observer-based robust control of attitude tracking of rigid spacecraft," Nonlinear Dynamics, vol. 88, no. 2, pp. 1317-1328, 2017.

[20] Z. Liu, J. Liu, and W. He, "Adaptive boundary control of a flexible manipulator with input saturation," International Journal of Control, vol. 89, no. 6, pp. 1191-1202, 2016.

[21] Z. Liu, J. Liu, and W. He, "Partial differential equation boundary control of a flexible manipulator with input saturation," International Journal of Systems Science, vol. 48, no. 1, pp. 53-62, 2017.

[22] L. Meirovitch, Fundamentals of Vibrations, Waveland Press, 2010.

[23] M. S. de Queiroz, D. M. Dawson, S. P. Nagarkatti, and F. Zhang, "Lyapunov-based control of mechanical systems," Applied Mechanics Reviews, vol. 54, no. 5, p. B81, 2001.

[24] L. Guo and S. Y. Cao, Anti-Disturbance Control for Systems with Multiple Disturbances, Crc Press, 2017.

[25] K. J. Astrom, Introduction To Stochastic Control Theory, Academic Press, New York, NY, USA, 1970.

[26] W. Chen, J. Yang, L. Guo, and S. Li, "Disturbance observer-based control and related methods: an overview," IEEE Transactions on Industrial Electronics, vol. 63, no. 2, pp. 1083-1095, 2016.

[27] K.-S. Kim, K.-H. Rew, and S. Kim, "Disturbance observer for estimating higher order disturbances in time series expansion," Institute of Electrical and Electronics Engineers Transactions on Automatic Control, vol. 55, no. 8, pp. 1905-1911, 2010. 


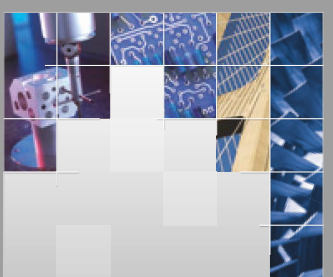

\section{Enfincering}
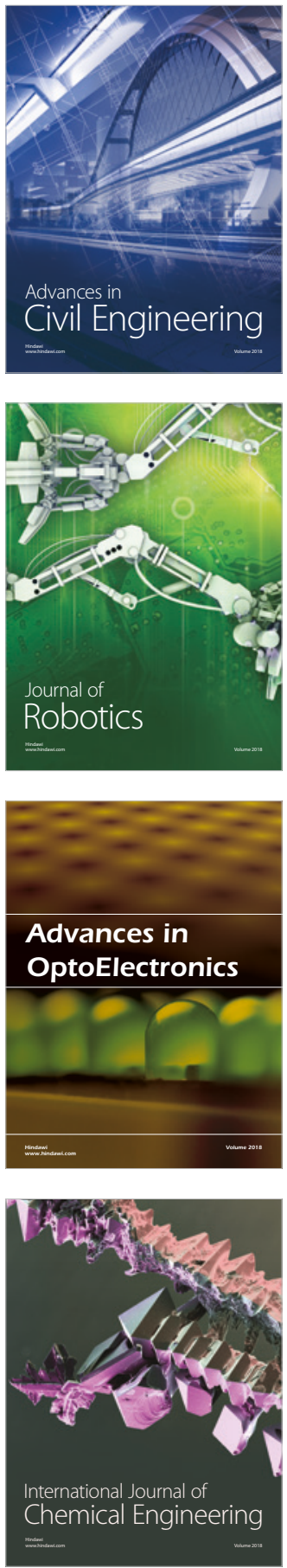

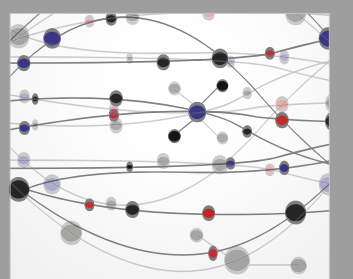

\section{Rotating \\ Machinery}

The Scientific World Journal

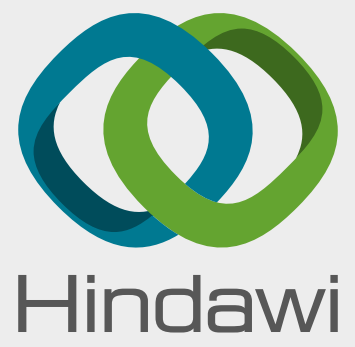

Submit your manuscripts at

www.hindawi.com
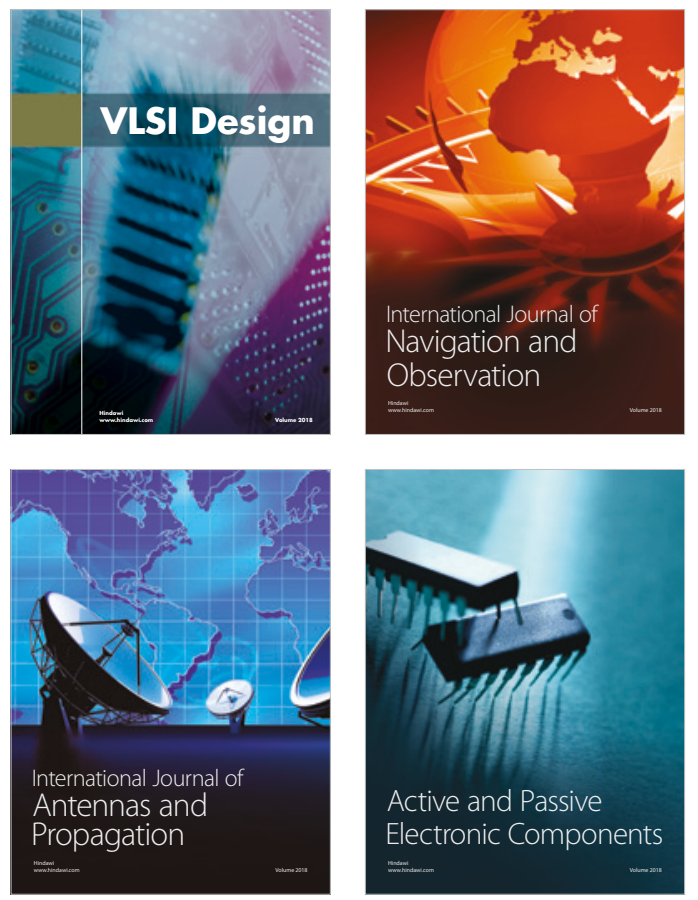
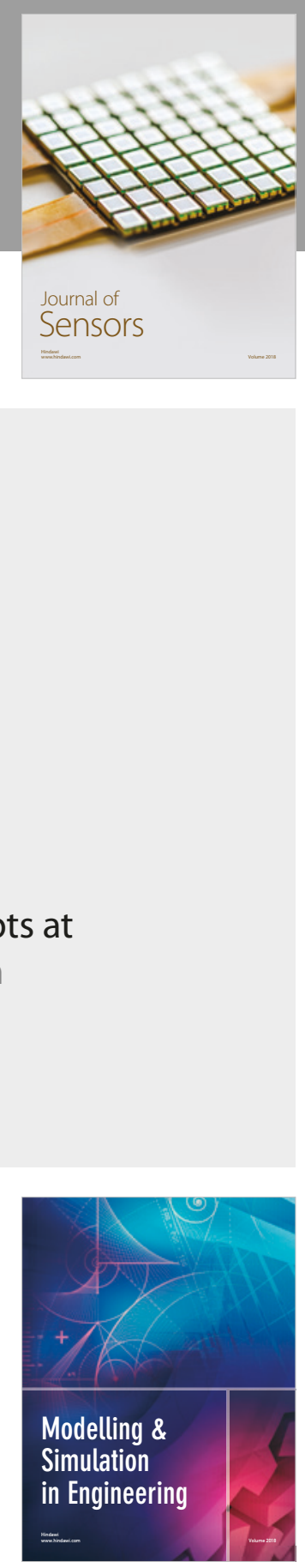

\section{Advances \\ Multimedia}
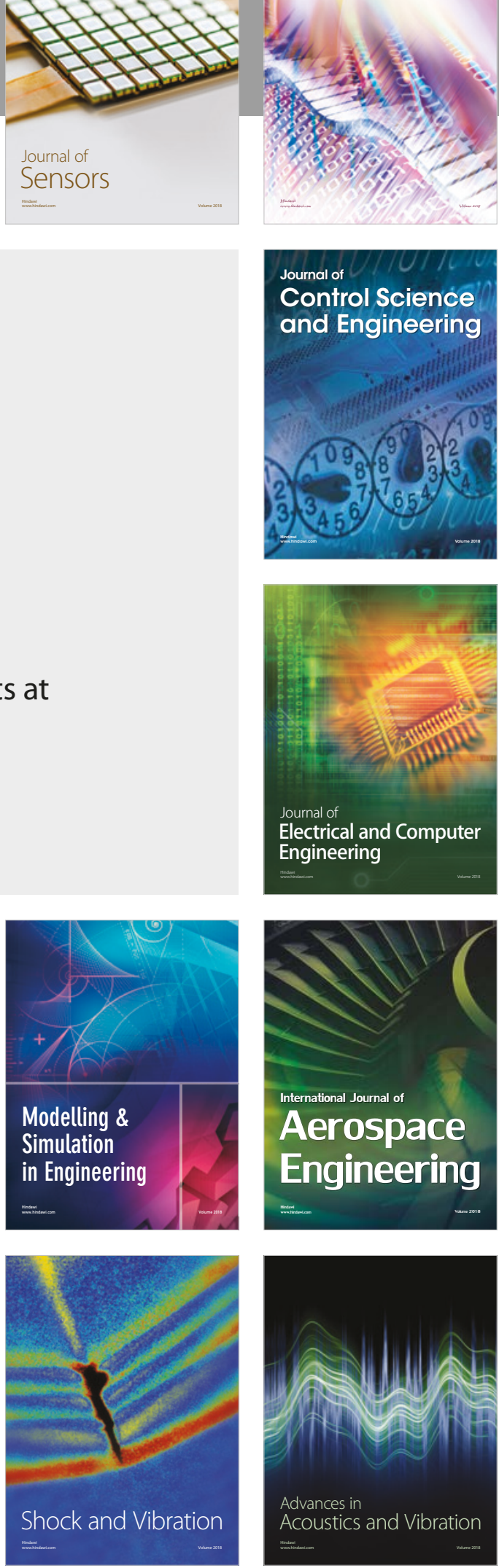